\title{
Practical monitoring of the clinical efficacy of sinquanon in patients on antibiotic therapy
}

\author{
Prof. Dr. Veselin Pavlov, MD, Dr. Konstantin Borisov \\ ENT Clinic at the University First Multi-Profile Hospital for Active Treatment - Sofia EAD
}

\begin{abstract}
Summary
For more than seven decades, antibiotics and chemotherapeutics have been used successfully to treat and prevent infectious diseases, extensively changing the prognosis of severe infections and helping to improve quality of life. In the last two decades, the key role of the intestinal microbiota (IM) in basic vital functions such as digestion, protection against pathogens, and maintenance of immune and metabolic homeostasis has been recognized. Antibiotics do not selectively attack pathogenic bacteria. They have an effect on the normal microflora and can lead to imbalance inside the body (dysbacteriosis) and the development of opportunistic infections. Taking a probiotic during antibiotic treatment is one of the approaches to prevent antibiotic-associated diarrhea and other adverse effects of antibiotics when their use is necessary. In the present practical monitoring we want to share our results from the application of Sinquanon ${ }^{\circledR}$ to neutralize the negative effect of antibiotic treatment on the microbiome.
\end{abstract}

\section{Introduction}

In daily otorhinolaryngological practice in the treatment of bacterial infections, the role of antibiotics is indispensable. However, when dealing with pathogenic bacteria, they have detrimental effects on the microflora, which can be clinically manifested both in the short and long term. Increasing scientific evidence supports the relationship between impaired gastrointestinal microflora and diseases outside the gastrointestinal tract, which may find clinical manifestation years after antibiotic administration. Intestinal dysbiosis is characterized by significant loss of beneficial bacteria, excessive growth of potential pathogens, loss of microbial diversity and reduced stability. One of the most common complications that immediately follow antibiotic therapy is antibiotic-associated diarrhea, which can affect more than $30 \%$ of patients. Irritable bowel syndrome is another complication that can occur immediately after the course of antibiotics and months, even years after.

We must emphasize the fact that probiotic products are not the same! Most of the existing probiotics are not closely specialized, so they cannot fully protect the intestinal microbiome during $\mathrm{AB}$ therapy, resulting in a number of adverse side effects of the antibiotic. Sinquanon ${ }^{\circledR}$ is a specialized probiotic designed to help all patients with $\mathrm{AB}$ treatment regardless of the type of $A B$ taken and whether the patient has had side effects from antibiotics in the past. 
Intake of Sinquanon ${ }^{\circledR}$ helps to restore the microbial diversity and amount lost during antibiotic treatment as quickly and completely as possible, restoring the microbiome to its initial levels, thus reducing the occurrence of antibiotic-associated diarrhea and the onset of pain/flatulence.

\section{Material and methods}

Our practical clinical monitoring was conducted in the ENT Slinic at the ENT Clinic at the University First Multi-Profile Hospital for Active Treatment-Sofia EAD, for the period 01.06.2020 31.07.2020 and covered 42 patients aged between 11 and 89 years with diagnoses: Abscessus peritonsillaris, Tonssilitis lacunaris, Epistaxis and Otitis media serosa acuta. For the purposes of the study, we compiled a protocol for Practical Clinical Monitoring. Of our patients, 8 were on parenteral and 34 on oral therapy, and in 34 of the cases the anti- biotic used was Amoxiclav 625, in 2 cases we used Unasyn and in 6 cases - Ceftriaxone.

Pre-hospital therapy was performed in 8 patients. No adverse effects of $\mathrm{AB}$ treatment were reported in 38 of 42 patients. Only 4 had side effects, with two experiencing abdominal pain and antibiotic associated diarrhea (AAD) on day 3, and had started taking Sinquanon ${ }^{\circledR}$ after the onset of complaints. Two patients showed AAD on day 2. From these data, we concluded that Sinquanon ${ }^{\circledR}$ provided a $90 \%$ reduction in the side effects of the applied $\mathrm{AB}$ treatment.

In a control group of 16 patients, for the same period of time, aged between 19 and 68 years, 4 of them showed symptoms of abdominal pain and AAD on the second day of antibiotic therapy. Flatulence was reported in 2 patients in the group on day 3 .

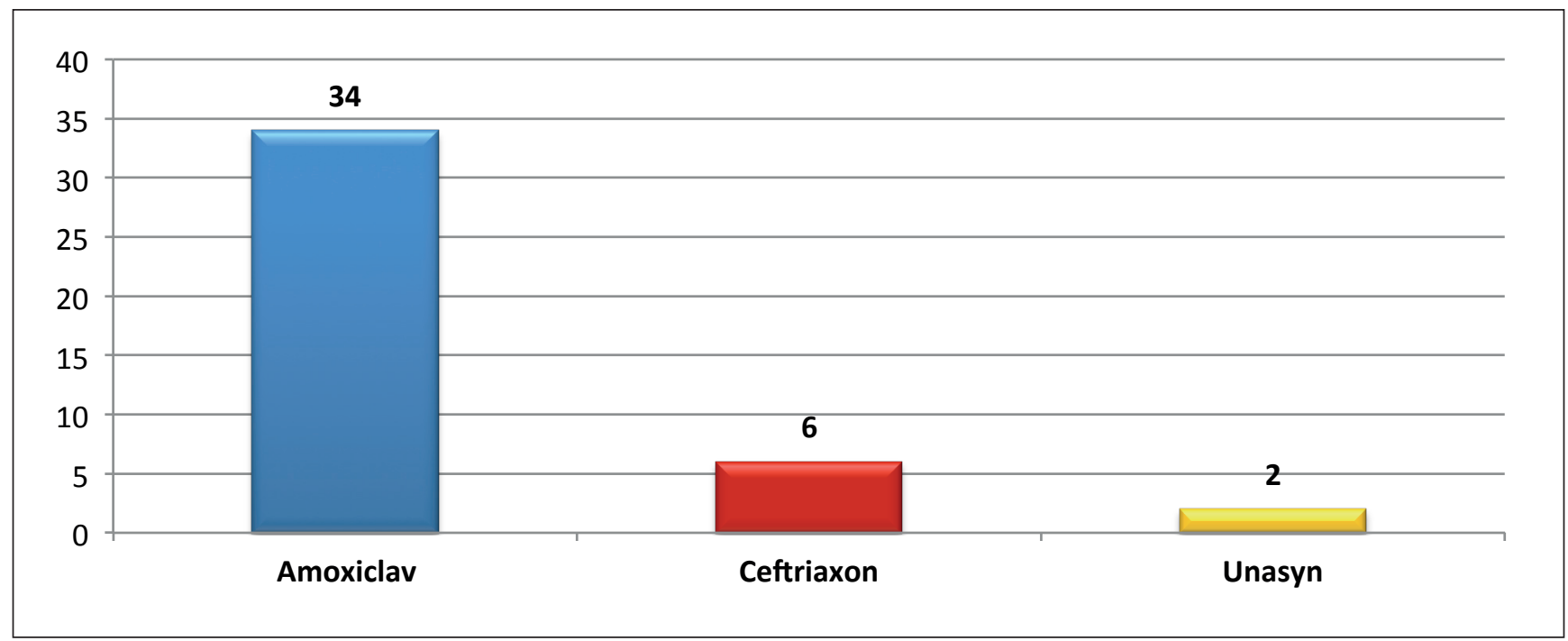

Fig 1. Alignment of of patients according to the type of antibiotic therapy

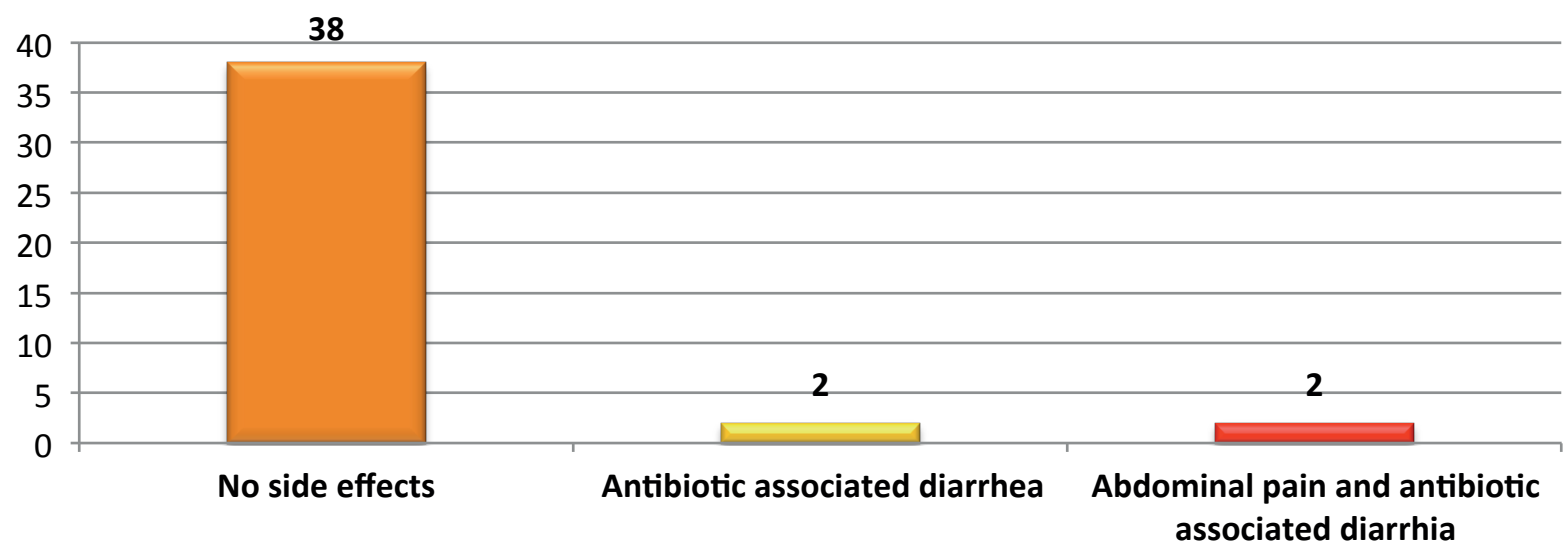

Fig 2. Clinical efficacy of Sinquanon ${ }^{\circledR}$ in patients on $A B$ therapy 


\section{Discussion}

Sinquanon ${ }^{\circledR}$ is a specialized probiotic for antibiotic therapy, which promptly helps to restore to the greatest extent the lost microbial count of beneficial bacteria in the intestinal microbiota, restores lost biodiversity in the intestinal ecosystem, helps the reproduction of beneficial bacteria and supplies additional $\mathrm{B}$ vitamins. It contains a mixture of 14 specially selected probiotic strains, in a daily dose of 100 billion living cells. It has been proven that the action of probiotics depends on the dose - the higher it is, the better the effect. The high number of beneficial bacteria collected in a daily dose makes Sinquanon ${ }^{\circledR}$ a specialized probiotic to be used with antibiotic. Secondly, what distinguishes it is the large strain diversity of beneficial bacteria contained in it, specially selected and fully tailored to the needs of patients on antibiotic treatment. It is important to take a probiotic product containing strains that will survive during AB therapy. Sinquanon $^{\circledR}$ contains strains with natural resistance against various $\mathrm{AB}$. The probiotic strains in Sinquanon ${ }^{\circledR}$ are of the genus Lactobacillus, Bifidobacterium, Saccharomyces, and spore-forming bacilli. They are characterized by a high rate of division and high natural resistance to antibiotics. The probiotic contains two of the best studied worldwide and highly recommended by the European Society for Paediatric Gastroenterology, Hepatology and Nutrition and the World Gastroenterology Organization probiotic strains for the prevention of antibiotic- associated diarrhea - Lactobacillus rhamnosus GG and Saccharomyces boulardii. Sinquanon ${ }^{\circledR}$ also contains a special probiotic strain Bacillus coagulans, which turns into a spore under adverse conditions. This makes it extremely resistant to harmful influences, for example in antibiotic therapy. It also increases the population of beneficial bacteria in the gut. The presence of so many different strains contributes to their synergistic action. During antibiotic treatment, the normal synthesis of B vitamins decreases, so in addition to the probiotic blend, Sinquanon ${ }^{\circledR}$ also contains a complex of six B vitamins in the recommended daily doses. It also contains a patented prebiotic blend, specially created for the needs of the 14 probiotic strains included in its composition. It includes three types of oligosaccharides that are not degradable by the human body, which serve as food for probiotic strains and beneficial bacteria that live normally in the gastrointestinal tract. Prebiotics help strains to divide even faster. The Sinquanon ${ }^{\circledR}$ capsule is gastro-resistant, which means that it passes through the aggressive stomach acid and dissolves in the small intestine. As a result of this, it delivers live probiotic bacteria to the right place, where they have their beneficial effect.

\section{References}

1. Petra Zimmermann, Nigel Curtis The effect of antibiotics on the composition of the intestinal microbiota - a systematic review Published:October 18, 2019

2. Xing Wang Gao, Dose - Response Effi cacy of a Proprietary Probiotic Formula of Lactobacillus acidophilus CL1285 and Lactobacillus casei LBC80R for Antibiotic-Associated Diarrhea and Clostridium difficile -Associated Diarrhea Prophylaxis in Adult Patients, MAm J Gastroenterol 2010; 105:1636-1641; doi: 10.1038/ajg.2010.11

3. Chapman, C.M.C., Gibson, G.R. \& Rowland, I., Health benefits of probiotics: are mixtures more effective than single strains?, Eur J Nutr (2011) 50: 1 .

4. ESPGHAN, Probiotics for the Prevention of Antibiotic-Associated Diarrhea in Children, JPGN Volume 62, Number 3, March 2016

5. WGO Global Guideline Probiotics and prebiotics, 2017 